\title{
Numerical Solutions of Duffing Equations Involving Linear Integral with Shifted Chebyshev Polynomials
}

\author{
Ayşe Anapalı, Yalçın ÖZTÜRK, Mustafa GÜLSU \\ Department of Mathematics, Faculty of Science, Muğla University, Muğla, Turkey \\ Ula Ali Koçman Vocational School, Muğla University, Muğla, Turkey \\ e-posta:yozturk@mu.edu.tr
}

Geliş Tarihi:14.11.2014; Kabul Tarihi:03.04.2015

\begin{tabular}{|c|c|}
\hline Keywords & Abstract \\
\hline $\begin{array}{l}\text { Duffing-van der Pol } \\
\text { equation; Duffing } \\
\text { equation; Shifted } \\
\text { Chebyshev } \\
\text { polynomials; } \\
\text { Chebyshev series; } \\
\text { Shifted Chebyshev } \\
\text { polynomial solutions; } \\
\text { Approximation }\end{array}$ & $\begin{array}{l}\text { The purpose of this study is to give a shifted Chebyshev polynomial approximation for the } \\
\text { solution of Duffing-van der Pol equation involving linear integral term (DEILI). For this } \\
\text { purpose, a new Chebyshev collocation method is introduced. This method is based on } \\
\text { taking the truncated shifted Chebyshev expansion of the function. This method based on } \\
\text { first taking the truncated Chebyshev series of the solution function in the DEILI and then, } \\
\text { transforms DEILI and given conditions into a matrix equation and then, we have the } \\
\text { system of nonlinear algebraic equation using collocation points. Then, solving the system } \\
\text { of algebraic equations we have the coefficients of the truncated Chebyshev series. In } \\
\text { addition, examples that illustrate the pertinent features of the method are presented, and } \\
\text { the results of study are discussed. }\end{array}$ \\
\hline
\end{tabular}

\section{Lineer İntegral Terim İçeren Duffing Denkleminin Shifted Chebyshev Polinomları ile Nümerik Çözümleri}

\footnotetext{
Anahtar kelimeler

Duffing-Van der Pol denklemi; Duffing

denklemi; Shifted

Chebyshev

Polinomları;

Chebyshev serisi;

Shifted Chebyshev

polinom çözümleri;

Yaklaşım metodu
}

\section{Özet}

Bu çalışmanın amacı linear terim içeren Duffing-van der Pol denkleminin shifted Chebyshev polinomları yardımı ile yaklaşık çözümlerini sunmaktır. Bu amaçla Chebyshev sıralama metodu verilmiştir. Metodun ana karekteristiği verilen denklemi kesilmiş Chebyshev serisinin katasyılarının içeren bir denklem sistemine indirgemesidir. Bu sistem çözülerek kesilmiş Chebyshev serisinin katsayıları bulunur. Dolayısıyla yaklaşık çözüm elde edilir. Ayrıca, metodun uygulanabilirlini göstermek için örnekler sunulmuştur.

\section{Introduction}

Duffing equation is a mathematical model to describe a classical oscillator in a double-well by a periodical driven, which has been widely investigated in chaotic phenomena (Mickens, 1981; Guckenheimer and Holmes, 1983). It arises in a variety of different scientific fields such as periodic orbit extraction, non-uniformity caused by an infinite domain, nonlinear mechanical oscillators, prediction of diseases (Ahmad and Alghamdi,2007; Tang, 1998). (Ahmad and Alghandi, 2008) presented the existence and uniquenes 
solution of the Duffing equation involving both integral and non-integral forcing terms with separated boundary conditions by a generalized quasilinearization technique. The numerical solutions of the Duffing equation with two-point boundary conditions have been investigated by many researchers (Yao,2009; Geng,2011; Geng,2009).

In this paper, we consider the Duffing equation involving linear integral, which can be written as

$$
\begin{aligned}
& y^{\prime \prime}(t)+\gamma y^{\prime}(t)+f\left(t, y, y^{\prime}\right) \\
& +\int_{0}^{t} k(t, s) y(s) d s=g(t) \quad 0<t<1
\end{aligned}
$$

with the initial conditions

$$
\lambda_{0} y(0)-\lambda_{1} y^{\prime}(0)=\alpha
$$

and $\mu_{0} y(1)+\mu_{1} y^{\prime}(1)=\beta$

where $\gamma, \lambda_{0}, \lambda_{1}, \mu_{0}, \mu_{1}, \alpha$ and $\beta$ are real constant.

The aim of this study is to get solution as truncated Chebyshev series defined by

$$
\begin{aligned}
y_{N}(t) & =\sum_{n=0}^{N} a_{n} T_{n}^{*}(t), \\
T_{n}^{*}(t) & =\cos (n \theta), 2 t-1=\cos \theta
\end{aligned}
$$

where $T_{n}^{*}(t) \quad$ denotes the shifted Chebyshev polynomials of the first kind; $\sum$ ' denotes a sum whose first term is halved; $a_{n}(0 \leq n \leq N)$ are unknown Chebyshev coefficients, and $\mathrm{N}$ is chosen any positive integer.

\section{Preliminaries and notations}

In this section, we state some basic results about polynomial approximations. These important properties will enable us to solve the Duffing equations. Polynomials are the only functions that the computer can evaluate exactly, so we make approximate functions $R \rightarrow R$ by polynomials. We consider real-valued functions on the compact interval $[a, b]$ :

$$
f:[a, b] \rightarrow R
$$

and we denote the set all real-valued polynomials on $[a, b]$ by $\mathrm{P}$, that is

$$
\forall p \in \mathrm{P}, \forall x \in[a, b], p(x)=\sum_{i=0}^{N} a_{i} x^{i}
$$

and

$$
\mathrm{P}_{N}=\left\{p(x): \operatorname{deg}(p(x)) \leq N, N \in Z^{+}\right\}
$$

The uniform norm (or maximum norm) is defined by

$$
\|f\|_{\infty}=\max _{x \in[a, b]}|f(x)| .
$$

Definition 2.1 For a given continuous function $f \in C[a, b], \quad$ a best approximation polynomial of degree $N$ is a polynomial $p_{N}^{*}(f) \in P_{N}$ such that

$$
\left\|f-p_{N}^{*}(f)\right\|_{\infty}=\min \left\{\|f-p\|_{\infty}: p \in P_{N}\right\}
$$

where the uniform norm is defined by

$$
\|f\|_{\infty}=\max _{x \in[a, b]}|f(x)| .
$$

Theorem 2.1 (Rivlin,1969; Davis,1963) Let $f \in C[a, b]$. Then for any $\varepsilon>0$, there exist a polynomial $p$ for which

$$
\|f-p\|_{\infty} \leq \varepsilon
$$

The theorem states that any continuous function $f$ can be approximated uniformly by polynomials, no matter how badly behaved $f$ may be on $[a, b]$. For phrasing; for any continuous function on $[a, b], f$, there exist a sequence of polynomial $\left(p_{N}\right)_{N \in N}$ which converges uniformly towards $f$ such that 


$$
\lim _{N \rightarrow \infty}\left\|f-p_{N}\right\|=0
$$

Theorem 2.2 (Rivlin,1969; Davis,1963; Boyd, 2000; Atkinson, 2009; Mason and Handscomb, 2003) For any $f \in[a, b]$ and $N \geq 0$ the best approximation polynomial $p^{*}{ }_{N}(f)$ exists and is unique.

Definition 2.2 Given an integer $N \geq 1$ a grid is a set of $(N+1)$ points

$X=\left(x_{i}\right)_{0 \leq i \leq N}$ in $[a, b]$ such that $a \leq x_{0}<x_{1}<\cdots<x_{N} \leq b$. Then points $\left(x_{i}\right)_{0 \leq i \leq N}$ are called the nodes of the grid.

Theorem 2.3 (Rivlin,1969; Davis,1963; Boyd, 2000; Atkinson, 2009; Mason and Handscomb, 2003) Given a function $f \in C[a, b]$ and a grid of $(N+1)$ nodes $X=\left(x_{i}\right)_{0 \leq i \leq N}$, there exist a unique polynomial $I_{N}^{X}(f)$ of degree $N$ such that

$$
I_{N}^{X}(f)\left(x_{i}\right)=f\left(x_{i}\right), 0 \leq i \leq N
$$

$I_{N}^{X}(f)$ is called the interpolant (or interpolating polynomial) of $f$ through the grid $X$. The interpolant $I_{N}^{X}(f)$ can be express in the Lagrange form:

$$
I_{N}^{X}(f)=\sum_{i=0}^{N} f\left(x_{i}\right) \ell_{i}^{X}(x)
$$

where $\ell_{i}^{X}(x)$ is the $\mathrm{i}$-th Lagrange cardinal polynomial associated with the grid $X$ :

$$
\ell_{i}^{X}(x)=\prod_{j=0, i \neq j}^{N} \frac{x-x_{i}}{x_{i}-x_{j}}, 0 \leq i \leq N .
$$

The Lagrange cardinal polynomials are such that

$$
\ell_{i}^{X}\left(x_{j}\right)=\delta_{i j}=\left\{\begin{array}{ll}
1 & i=j \\
0 & i \neq j
\end{array}, 0 \leq i, j, \leq N .\right.
$$

The best approximation polynomials $p_{N}^{*}(f)$ are also an interpolant of $f$ at $N+1$ nodes and the error in given by :

$\left\|f-I_{N}^{X}(f)\right\|_{\infty} \leq\left(1+\Lambda_{N}(X)\right)\left\|f-p_{N}^{*}(f)\right\|_{\infty}$ where $\Lambda_{N}(X)$ is the Lebesque constant relative to the grid $X$

$$
\Lambda_{N}(X):=\max _{x \in[a, b]} \sum_{i=0}^{N}\left|\ell_{i}^{X}(x)\right|
$$

The Lebesque constant contains all the information on the effects of the choice of $X$ on $\left\|f-I_{N}^{X}(f)\right\|_{\infty}$.

Theorem 2.4 (Rivlin,1969; Davis,1963; Boyd, 2000; Atkinson, 2009; Mason and Handscomb, 2003) For any choice of the grid $X$, there exist a constant $C>0$ such that

$\Lambda_{N}(x)>\frac{2}{\pi} \ln (N+1)-C$.

Corollary 2.1 (Rivlin,1969; Davis,1963; Boyd, 2000; Atkinson, 2009; Mason and Handscomb, 2003) Let $\Lambda_{N}(X)$ be Lebesque constant relative to the grid $\mathrm{X}$, then

$\Lambda_{N}(X) \rightarrow \infty$ as $n \rightarrow \infty$.

In a similar way, by a uniform grid,

$\Lambda_{N}(X) \sim \frac{2^{N+1}}{e N \ln N}$ as $N \rightarrow \infty$.

This means that for any choice of type sampling of $[a, b]$, there exists a continuous function $f \in C[a, b]$ such that $I_{N}^{X}(f)$ does not convergence uniformly towards $f$. Let assume that the function $f$ is sufficiently smooth to have derivatives at least up to order $(N+1)$, with $f^{(N+1)}$ continuous i.e. $f \in C^{N+1}[a, b]$. 
Definition 2.3 The nodal polynomial associated with the grid is the unique polynomial of degree $(N+1)$ and leading coefficient 1 whose zeroes are the $(N+1)$ nodes of $X$ :

$$
w_{N+1}^{X}(x)=\prod_{i=0}^{N}\left(x-x_{i}\right) \text {. }
$$

Theorem 2.5 (Rivlin,1969; Davis,1963; Boyd, 2000; Atkinson, 2009; Mason and Handscomb, 2003) If $f \in C^{N+1}[a, b]$, then for any grid $X$ of $(N+1)$ nodes, and for any $x \in[a, b]$, the interpolation error is

$$
f(x)-I_{N}^{X}(f)(x)=\frac{f^{(N+1)}(\varsigma)}{(N+1) !} w_{N+1}^{X}(x)
$$

where $\varsigma=\varsigma(x) \in[a, b]$ and $w_{N+1}^{X}(x)$ nodal polynomial associated with the grid $X$.

Definition 2.5 The grid $X=\left(x_{i}\right)_{0 \leq i \leq N}$ such that the $x_{i}$ 's are the $(N+1)$ zeroes of the Chebyshev polynomial of degree $(N+1)$ is called the Chebyshev-Gauss (CG) grid.

Theorem 2.7 (Rivlin,1969; Davis,1963; Boyd, 2000; Atkinson, 2009; Mason and Handscomb, 2003) The polynomials of degree $(N+1)$ and leading coefficient 1 , the unique polynomial which has the smallest uniform norm on $[a, b]$ is the $(n+1)$ th Chebyshev polynomial divided by $2^{N}$.

\subsection{Chebyshev polynomials}

Definition 2.11 The Chebyshev polynomials $T_{n}(x)$ of the first kind is a polynomials in $x$ of degree $n$, defined by relation (Mason and Handscomb,2003)

$T_{n}(x)=\cos n \theta$, when $x=\cos \theta$

If the range of the variable $x$ is the interval $[-1,1]$, the range the corresponding variables $\theta$ can be taken $[0, \pi]$. We map the independent variable $x$ in $[0,1]$ to the variable $s$ in $[-1,1]$ by transformation

$s=2 x-1$ or $x=\frac{1}{2}(s+1)$

and this lead to the shifted Chebyshev polynomial of the first kind $T_{n}^{*}(x)$ of degree $n$ in $x$ on [0,1] given by [13]

$T_{n}^{*}(x)=T_{n}(s)=T_{n}(2 x-1)$.

It is of course possible to defined $T_{n}^{*}(x)$, like $T_{n}(x)$, directly by a trigonometric relation. Indeed, we obtained

$T_{n}^{*}(x)=\cos 2 n \theta$ when $x=\cos ^{2} \theta$.

The leading coefficient of $x^{n}$ in $T_{n}^{*}(x)$ to be $2^{2 n-1}$. These polynomials have the following properties (Mason and Handscomb,2003):

i) $T^{*}{ }_{n+1}(x)$ has exactly $n+1$ real zeroes on the interval $[0,1]$. The $i$-th zero $x_{n, i}$ of ,for $i=0,1, \ldots, n$

$x_{n, i}=\frac{1}{2}\left(1+\cos \left(\frac{(2(n-i)+1) \pi}{2(n+1)}\right)\right)$

ii) It is well known that the relation between the powers $x^{n}$ and the second kind Chebyshev polynomials $T_{n}^{*}(x)$ is for $0 \leq x \leq 1$,

$x^{n}=2^{-2 n+1} \sum_{k=0}^{n}\left(\begin{array}{l}2 n \\ k\end{array}\right) T_{n-k}^{*}(x)$

where $\sum$ ' denotes a sum whose first term is halved.

\section{Fundamental relations}

In this section, we give the matrix forms of each term in the Eq.(1) and conditions. 


\subsection{Matrix representation of the differential part}

We consider the solution $y(x)$ of Eq. (1) and its derivative $y_{N}{ }^{(k)}(x)$ defined by a truncated Chebyshev series (3).Then, we can put series in the matrix form, for $k=0,1,2$

$y(t)=\mathbf{T}^{*}(t) \mathbf{A}, \quad y^{(k)}(t)=\mathbf{T}^{*(k)}(t) \mathbf{A}$

where

$\mathbf{T}^{*}(t)=\left[T_{0}^{*}(t) T_{1}^{*}(t) \ldots T_{N}^{*}(t)\right]$

$\mathbf{A}=\left[\begin{array}{llll}a_{0} & a_{1} & \ldots & a_{N}\end{array}\right]^{T}$

By using the expression (5) and taking $\mathrm{n}=0,1, \ldots, \mathrm{N}$ we find the corresponding matrix relation as follows

$$
\begin{aligned}
& (\mathbf{X}(t))^{T}=\mathbf{D}\left(\mathbf{T}^{*}(t)\right)^{T} \text { and } \\
& \mathbf{X}(t)=\mathbf{T}^{*}(t) \mathbf{D}^{T}
\end{aligned}
$$

where

$$
\mathbf{X}(t)=\left[\begin{array}{lll}
1 & t \ldots t^{N}
\end{array}\right]
$$

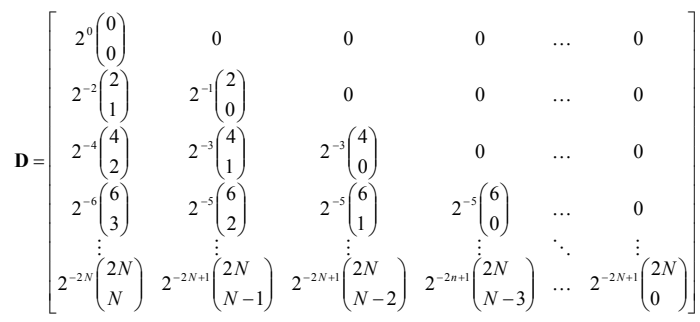

Then, by taking into account (7) we obtain

$$
\mathbf{T}^{*}(t)=\mathbf{X}(t)\left(\mathbf{D}^{-1}\right)^{T}
$$

and

$$
\left(\mathbf{T}^{*}(t)\right)^{(k)}=\mathbf{X}^{(k)}(t)\left(\mathbf{D}^{-1}\right)^{T}, \quad k=0,1,2
$$

To obtain the matrix $\mathbf{X}^{(k)}(t)$ in terms of the matrix $\mathbf{X}(t)$, we can use the following relation:

$$
\begin{aligned}
& \mathbf{X}^{(1)}(t)=\mathbf{X}(t) \mathbf{B}^{T} \\
& \mathbf{X}^{(2)}(t)=\mathbf{X}^{(1)}(t) \mathbf{B}^{T}=\mathbf{X}(t)\left(\mathbf{B}^{T}\right)^{2}
\end{aligned}
$$

where

$$
\mathbf{B}=\left[\begin{array}{ccccc}
0 & 0 & 0 & \ldots & 0 \\
1 & 0 & 0 & \ldots & 0 \\
0 & 2 & 0 & \ldots & 0 \\
\ldots & \ldots & \ldots & \ldots & \ldots \\
0 & 0 & 0 & N & 0
\end{array}\right]
$$

Consequently, by substituting the matrix forms (8) and (9) into (6) we have the matrix relation for $k=0,1,2$

$$
y^{(k)}=\mathbf{X}(t) \mathbf{B}^{k}\left(\mathbf{D}^{T}\right)^{-1} \mathbf{A}
$$

Moreover, substituting the zeroes of Chebyshev polynomials in Eq.(4) into Eq. (6), we have

$$
\begin{aligned}
& y\left(t_{i}\right)=\mathbf{T}\left(t_{i}\right) \mathbf{A} \text { and } \\
& y^{(k)}\left(t_{i}\right)=\mathbf{T}^{(k)}\left(t_{i}\right) \mathbf{A} \\
& =\mathbf{X}\left(t_{i}\right) \mathbf{B}^{k}\left(\mathbf{D}^{T}\right)^{-1} \mathbf{A}
\end{aligned}
$$

or compact form

$$
\mathbf{Y}=\mathbf{T A} \text { and }
$$

$$
\begin{aligned}
& \mathbf{Y}^{(k)}\left(t_{i}\right)=\mathbf{T}^{(k)}\left(t_{i}\right) \mathbf{A} \\
& =\mathbf{X B}^{k}\left(\mathbf{D}^{T}\right)^{-1} \mathbf{A}
\end{aligned}
$$

where

$$
\mathbf{T}=\left[\begin{array}{c}
\mathbf{T}\left(t_{0}\right) \\
\mathbf{T}\left(t_{1}\right) \\
\vdots \\
\mathbf{T}\left(t_{N}\right)
\end{array}\right] \mathbf{Y}=\left[\begin{array}{c}
y\left(t_{0}\right) \\
y\left(t_{1}\right) \\
\vdots \\
y\left(t_{N}\right)
\end{array}\right]
$$

$$
\mathbf{Y}^{(k)}=\left[\begin{array}{c}
y^{(k)}\left(t_{0}\right) \\
y^{(k)}\left(t_{1}\right) \\
\vdots \\
y^{(k)}\left(t_{N}\right)
\end{array}\right]
$$




$$
\mathbf{X}=\left[\begin{array}{ccccc}
1 & x_{0} & x_{0}^{2} & \cdots & x_{0}{ }^{N} \\
1 & x_{1} & x_{1}^{2} & \cdots & x_{1}{ }^{N} \\
1 & x_{2} & x_{2}^{2} & \cdots & x_{2}{ }^{N} \\
\vdots & \vdots & \vdots & \ddots & \vdots \\
1 & x_{N} & x_{N}^{2} & \cdots & x_{N}{ }^{N}
\end{array}\right]
$$

Similarly, substituting the zeroes of Chebyshev polynomial into $y^{r}(t)$, we obtained the matrix representation

$$
\mathbf{Y}^{r}=(\overline{\mathbf{Y}})^{r-1} \mathbf{Y}
$$

where

$$
\begin{aligned}
& \mathbf{Y}^{r}=\left[\begin{array}{c}
y^{r}\left(t_{0}\right) \\
y^{r}\left(t_{1}\right) \\
\vdots \\
y^{r}\left(t_{N}\right)
\end{array}\right] \\
& \overline{\mathbf{Y}}=\left[\begin{array}{cccc}
y\left(t_{0}\right) & 0 & \cdots & 0 \\
0 & y\left(t_{1}\right) & \cdots & 0 \\
\vdots & \vdots & \ddots & \vdots \\
0 & 0 & \cdots & y\left(t_{N}\right)
\end{array}\right]
\end{aligned}
$$

and

$$
\overline{\mathbf{Y}}=\overline{\mathbf{T}} \overline{\mathbf{A}}
$$

where

$$
\overline{\mathbf{T}}=\left[\begin{array}{cccc}
\mathbf{T}\left(t_{0}\right) & 0 & \cdots & 0 \\
0 & \mathbf{T}\left(t_{1}\right) & \cdots & 0 \\
\vdots & \vdots & \ddots & \vdots \\
0 & 0 & \cdots & \mathbf{T}\left(t_{N}\right)
\end{array}\right]
$$$$
\overline{\mathbf{A}}=\left[\begin{array}{cccc}
\mathbf{A} & 0 & \cdots & 0 \\
0 & \mathbf{A} & \cdots & 0 \\
\vdots & \vdots & \ddots & \vdots \\
0 & 0 & \cdots & \mathbf{A}
\end{array}\right]
$$

We can write

$$
\begin{aligned}
f\left(t, y, y^{\prime}\right)= & \sum_{m=0}^{r} P_{m}(t) y^{m}(t) \\
& +\sum_{n=0}^{s} H_{n}(t) y^{n}(t) y^{\prime}(t)
\end{aligned}
$$

For obtained matrix form of $y^{m}, m \in Z^{+}$ and $y^{n} y^{\prime}, n \in Z^{+}$, using relation (14) and (15), we construct the following relations

$$
\begin{aligned}
& y^{2}\left(t_{i}\right)=y\left(t_{i}\right) y\left(t_{i}\right)=(\overline{\mathbf{T}} \overline{\mathbf{A}}) \mathbf{X}\left(t_{i}\right)\left(\mathbf{D}^{T}\right)^{-1} \mathbf{A} \\
& y^{3}\left(t_{i}\right)=y^{2}\left(t_{i}\right) y\left(t_{i}\right)=(\overline{\mathbf{T}} \overline{\mathbf{A}})^{2} \mathbf{X}\left(t_{i}\right)\left(\mathbf{D}^{T}\right)^{-1} \mathbf{A}
\end{aligned}
$$

$$
y^{m}\left(t_{i}\right)=y^{m-1}\left(t_{i}\right) y\left(t_{i}\right)=(\overline{\mathbf{T}} \overline{\mathbf{A}})^{m-1} \mathbf{X}\left(t_{i}\right)\left(\mathbf{D}^{T}\right)^{-1} \mathbf{A}
$$

and

$$
\begin{aligned}
& y\left(t_{i}\right) y^{\prime}\left(t_{i}\right)=(\overline{\mathbf{T}} \overline{\mathbf{A}}) \mathbf{X}\left(t_{i}\right) \mathbf{B}\left(\mathbf{D}^{T}\right)^{-1} \mathbf{A} \\
& y^{2}\left(t_{i}\right) y^{\prime}\left(t_{i}\right)=(\overline{\mathbf{T}} \overline{\mathbf{A}})^{2} \mathbf{X}\left(t_{i}\right) \mathbf{B}\left(\mathbf{D}^{T}\right)^{-1} \mathbf{A} \\
& \vdots \\
& y^{n}\left(t_{i}\right) y^{\prime}\left(t_{i}\right)=(\overline{\mathbf{T}} \overline{\mathbf{A}})^{n} \mathbf{X}\left(t_{i}\right) \mathbf{B}\left(\mathbf{D}^{T}\right)^{-1} \mathbf{A}
\end{aligned}
$$

Thus we can write

$$
\begin{aligned}
& f\left(x, y, y^{\prime}\right)=\sum_{m=0}^{r} \mathbf{P}_{m}(t)(\overline{\mathbf{T}} \overline{\mathbf{A}})^{m-1} \mathbf{X}\left(t_{i}\right)\left(\mathbf{D}^{T}\right)^{-1} \mathbf{A} \\
& +\sum_{n=0}^{s} \mathbf{H}_{n}(t)(\overline{\mathbf{T}} \overline{\mathbf{A}})^{n} \mathbf{X}\left(t_{i}\right) \mathbf{B}\left(\mathbf{D}^{T}\right)^{-1} \mathbf{A}
\end{aligned}
$$

\subsection{Matrix representation of integral part}

Let assume that $K(t, s)$ can be expanded to univariate Chebyshev series with respect to $t$ as follows:

$$
K(t, s)=\sum_{r=0}^{N} f_{s r}(t) T_{r}(s) .
$$

Then the matrix representations of the kernel function $K_{s}(x, t)$ become

$$
K(t, s)=\mathbf{F}(t) \mathbf{T}^{T}(s),
$$


where

$\mathbf{F}(t)=\left[\begin{array}{lllll}f_{0}(t) & f_{1}(t) & f_{2}(t) & \cdots & f_{N}(t)\end{array}\right]$.

Substituting the relations (6), (15) and (18) in integral part, we obtained

$$
\begin{aligned}
& I(t)=\int_{0}^{t} \mathbf{F}(t) \mathbf{T}^{T}(s) \mathbf{T}(s) \mathbf{A} d s \\
& =\int_{0}^{t} \mathbf{F}(t) \mathbf{D}^{-1} \mathbf{X}^{T}(s) \mathbf{X}(s)\left(\mathbf{D}^{T}\right)^{-1} \mathrm{~A} d s \\
& =\mathbf{F}(t) \mathbf{D}^{-1}\left(\int_{0}^{t} \mathbf{X}^{T}(s) \mathbf{X}(s) d s\right)\left(\mathbf{D}^{T}\right)^{-1} \mathbf{A} \\
& =\mathbf{F}(t) \mathbf{D}^{-1} \mathbf{Q}\left(\mathbf{D}^{T}\right)^{-1} \mathbf{A}
\end{aligned}
$$

where

$$
\mathbf{Q}=\int_{0}^{t} \mathbf{X}^{T}(s) \mathbf{X}(s) d s,
$$

and

$$
\mathbf{Q}=\left[q_{i j}\right]=\frac{t^{i+j+1}}{i+j+1}, \quad i, j=0,1, \ldots, N .
$$

\subsection{Matrix representation of conditions}

On the other hand, the matrix form for conditions (2) can be written as

$$
\begin{aligned}
& \lambda_{0} y(0)-\lambda_{1} y^{\prime}(0)= \\
& {\left[\lambda_{0} \mathbf{X}(0)\left(\mathbf{D}^{T}\right)^{-1}-\lambda_{1} \mathbf{X}(0) \mathbf{B}\left(\mathbf{D}^{T}\right)^{-1}\right] \mathbf{A}} \\
& \equiv\left[\begin{array}{llll}
u_{00} & u_{01} & \cdots & u_{0 N}
\end{array}\right] \mathbf{A}=[a]
\end{aligned}
$$

and

$$
\begin{aligned}
& \mu_{0} y(0)-\mu_{1} y^{\prime}(0)= \\
& {\left[\mu_{0} \mathbf{X}(1)\left(\mathbf{D}^{T}\right)^{-1}+\mu_{1} \mathbf{X}(1) \mathbf{B}\left(\mathbf{D}^{T}\right)^{-1}\right] \mathbf{A}} \\
& \equiv\left[\begin{array}{llll}
u_{10} & u_{11} & \cdots & u_{1 N}
\end{array}\right] \mathbf{A}=[b]
\end{aligned}
$$

\section{Method of solution}

Firstly, we construct the fundamental matrix equation corresponding to Eq.(1). For computing the Chebyshev coefficient matrix A numerically, the zeros of the Chebyshev polynomial defined by in Eq.(4) are put into the matrix form of Eq.(1). We obtain

$$
\begin{aligned}
& \mathbf{X}\left(t_{i}\right) \mathbf{B}^{2}\left(\mathbf{D}^{T}\right)^{-1}+\gamma \mathbf{X}\left(t_{i}\right) \mathbf{B}\left(\mathbf{D}^{T}\right)^{-1} \\
& +\sum_{m=0}^{r} \mathbf{P}_{m}\left(t_{i}\right)(\overline{\mathbf{T}} \overline{\mathbf{A}})^{m-1} \mathbf{X}\left(t_{i}\right)\left(\mathbf{D}^{T}\right)^{-1} \mathbf{A} \\
& +\sum_{n=0}^{s} \mathbf{H}_{n}\left(t_{i}\right)(\overline{\mathbf{T}} \overline{\mathbf{A}})^{n} \mathbf{X}\left(t_{i}\right) B\left(\mathbf{D}^{T}\right)^{-1} \mathbf{A} \\
& +\mathbf{F}\left(t_{i}\right) \mathbf{D}^{-1} \mathbf{Q}\left(\mathbf{D}^{T}\right)^{-1} \mathbf{A}=g\left(t_{i}\right)
\end{aligned}
$$

and then system can be written in the matrix form

$$
\begin{aligned}
& {\left[\mathbf{X B}^{2}\left(\mathbf{D}^{T}\right)^{-1}+\mathbf{E X B}\left(\mathbf{D}^{T}\right)^{-1}\right.} \\
& +\sum_{m=0}^{r} \mathbf{P}_{m}(\overline{\mathbf{T}} \overline{\mathbf{A}})^{m-1} \mathbf{X}\left(\mathbf{D}^{T}\right)^{-1} \\
& +\sum_{n=0}^{s} \mathbf{H}_{n}(\overline{\mathbf{T}} \overline{\mathbf{A}})^{n} \mathbf{X B}\left(\mathbf{D}^{T}\right)^{-1} \\
& \left.+\mathbf{F} \overline{\mathbf{D}^{-1}} \overline{\mathbf{Q}} \overline{\left(\mathbf{D}^{T}\right)^{-1}}\right] \mathbf{A}=\mathbf{G}
\end{aligned}
$$

where

$$
\mathbf{E}=\left[\begin{array}{ccccc}
\gamma & 0 & 0 & \cdots & 0 \\
0 & \gamma & 0 & \cdots & 0 \\
0 & 0 & \gamma & \cdots & 0 \\
\vdots & \vdots & \vdots & \ddots & \vdots \\
0 & 0 & 0 & \cdots & \gamma
\end{array}\right]
$$

$$
\mathbf{P}_{m}=\left[\begin{array}{ccccc}
\mathbf{P}_{m}\left(t_{0}\right) & 0 & 0 & \cdots & 0 \\
0 & \mathbf{P}_{m}\left(x_{t 1}\right) & 0 & \cdots & 0 \\
0 & 0 & \mathbf{P}_{m}\left(t_{2}\right) & \cdots & 0 \\
\vdots & \vdots & \vdots & \ddots & \vdots \\
0 & 0 & 0 & \cdots & \mathbf{P}_{m}\left(t_{N}\right)
\end{array}\right]
$$




$$
\begin{aligned}
& \mathbf{H}=\left[\begin{array}{ccccc}
\mathbf{H}_{n}\left(t_{0}\right) & 0 & 0 & \cdots & 0 \\
0 & \mathbf{H}_{n}\left(t_{1}\right) & 0 & \cdots & 0 \\
0 & 0 & \mathbf{H}_{n}\left(t_{2}\right) & \cdots & 0 \\
\vdots & \vdots & \vdots & \ddots & \vdots \\
0 & 0 & 0 & \cdots & \mathbf{H}_{n}\left(t_{N}\right)
\end{array}\right] \\
& \mathbf{F}=\left[\begin{array}{ccccc}
\mathbf{F}\left(t_{0}\right) & 0 & 0 & \cdots & 0 \\
0 & \mathbf{F}\left(t_{1}\right) & 0 & \cdots & 0 \\
0 & 0 & \mathbf{F}\left(t_{2}\right) & \cdots & 0 \\
\vdots & \vdots & \vdots & \ddots & \vdots \\
0 & 0 & 0 & \cdots & \mathbf{F}\left(t_{N}\right)
\end{array}\right] \\
& \overline{\mathbf{D}^{-1}}=\left[\begin{array}{ccccc}
\mathbf{D}^{-1} & 0 & 0 & \cdots & 0 \\
0 & \mathbf{D}^{-1} & 0 & \cdots & 0 \\
0 & 0 & \mathbf{D}^{-1} & \cdots & 0 \\
\vdots & \vdots & \vdots & \ddots & \vdots \\
0 & 0 & 0 & \cdots & \mathbf{D}^{-1}
\end{array}\right] \\
& \mathbf{Q}=\left[\begin{array}{ccccc}
\mathbf{Q}\left(t_{0}\right) & 0 & 0 & \cdots & 0 \\
0 & \mathbf{Q}\left(t_{1}\right) & 0 & \cdots & 0 \\
0 & 0 & \mathbf{Q}\left(t_{2}\right) & \cdots & 0 \\
\vdots & \vdots & \vdots & \ddots & \vdots \\
0 & 0 & 0 & \cdots & \mathbf{Q}\left(t_{N}\right)
\end{array}\right] \\
& \left(\overline{\left.\mathbf{D}^{T}\right)^{-1}}=\left[\begin{array}{c}
\left(\mathbf{D}^{T}\right)^{-1} \\
\left(\mathbf{D}^{T}\right)^{-1} \\
\left(\mathbf{D}^{T}\right)^{-1} \\
\vdots \\
\left(\mathbf{D}^{T}\right)^{-1}
\end{array}\right] \mathbf{G}=\left[\begin{array}{c}
g\left(t_{0}\right) \\
g\left(t_{1}\right) \\
g\left(t_{2}\right) \\
\vdots \\
g\left(t_{N}\right)
\end{array}\right]\right.
\end{aligned}
$$

Hence, the fundamental matrix equation (23) corresponding to Eq. (1) can be written in the form

$$
\begin{aligned}
& \mathbf{W A}=\mathbf{G} \text { or }[\mathbf{W} ; \mathbf{G}] \\
& \mathbf{W}=\left[w_{i, j}\right], i, j=0,1, \ldots, N
\end{aligned}
$$

where

$$
\begin{aligned}
& \mathbf{W}=\mathbf{X B}^{2}\left(\mathbf{D}^{T}\right)^{-1}+\mathbf{E X B}\left(\mathbf{D}^{T}\right)^{-1} \\
& +\sum_{m=0}^{r} \mathbf{P}_{m}(\overline{\mathbf{T}} \overline{\mathbf{A}})^{m-1} \mathbf{X}\left(\mathbf{D}^{T}\right)^{-1} \\
& +\sum_{n=0}^{s} \mathbf{H}_{n}(\overline{\mathbf{T}} \overline{\mathbf{A}})^{n} \mathbf{X B}\left(\mathbf{D}^{T}\right)^{-1}+\mathbf{F} \overline{\mathbf{D}^{-1}} \overline{\mathbf{Q}} \overline{\left(\mathbf{D}^{T}\right)^{-1}}
\end{aligned}
$$

To obtain the solution of Eq. (1) under conditions (2), by replacing the row matrices (21a)-(21b) by the last 2 rows of the matrix (24), we have the new augmented matrix,

$[\tilde{\mathbf{W}} ; \tilde{\mathbf{G}}]=$

$\left[\begin{array}{cccccc}w_{00} & w_{01} & \cdots & w_{0 N} & ; & f\left(t_{0}\right) \\ w_{10} & w_{11} & \cdots & w_{1 N} & ; & f\left(t_{1}\right) \\ \vdots & \vdots & \ddots & \vdots & \vdots & \vdots \\ w_{N-20} & w_{N-21} & \cdots & w_{N-2 N} & \vdots & f\left(t_{N-2}\right) \\ u_{00} & u_{01} & \cdots & u_{0 N} & ; & a \\ u_{10} & u_{11} & \cdots & u_{1 N} & ; & b\end{array}\right]$

So, we obtain a system of $(N+1)$ nonlinear algebraic equations with unknown shifted Chebyshev coefficients. Thus, we obtain the Chebyshev polynomial solution.

We can easily check the accuracy of the method. Since the truncated shifted Chebyshev series (3) is an approximate solution of Eq.(1), when the solution $y_{N}(t)$ and its derivatives are substituted in Eq.(1), the resulting equation must be satisfied approximately; that is (Body, 2000) for $t=t_{q} \in[0,1], \quad q=0,1,2, \ldots$

$$
\begin{aligned}
& E\left(x_{q}\right)=\mid y^{\prime \prime}\left(t_{q}\right)+\gamma y^{\prime}\left(t_{q}\right)+f\left(t_{q}, y, y^{\prime}\right) \\
& +\int_{0}^{t_{q}} k\left(t_{q}, s\right) y(s) d s-g\left(t_{q}\right) \mid \cong 0
\end{aligned}
$$

\subsection{Error analysis and convergence}

Since, $\left\|T_{N+1}^{*}\right\|_{\infty}=1$, we conclude that if we choose the grid nodes $\left(x_{i}\right)_{0 \leq i \leq N}$ to be zero the $(\mathrm{N}+1)$ zeroes of the Chebyshev polynomials $T_{N+1}^{*}$, we have 


$$
\left\|w_{N+1}^{X}\right\|=\frac{1}{2^{2 N-1}}
$$

and this is the smallest possible value. In particular, from Theorem 2.5, for any $y \in C^{N+1}[0,1]$ we have (Rivlin,1969; Davis,1963; Body,2000)

$\left\|y-y_{N}\right\|_{\infty} \leq \frac{1}{2^{2 N-1}(N+1) !}\left\|y^{N+1}\right\|_{\infty}$

If $y^{(N+1)}$ is uniformly bounded, the convergence of the interpolation $y_{N}$ towards $y$ when $N \rightarrow \infty$ is then extremely fast. Also the Lebesgue constant associated with the Chebyshev-Gauss grid is small

$$
\Lambda_{N}(X) \sim \frac{2}{\pi} \ln (N+1) \text { as } N \rightarrow \infty
$$

This is much better than uniform grids and close to the optimal value.

\section{Illustrative example}

In this section, several numerical examples are given to illustrate the accuracy and effectiveness properties of the method and all of them were performed on the computer using a program written in Maple 13.

Example 1 Consider the following Duffing equation involving linear integral term for $0<t<1$,

$$
y^{\prime \prime}+y^{\prime}+y\left(1+y^{\prime}\right)+\int_{0}^{t} s^{2} y(s) d s=g(t)
$$

with

$2 y(0)-y^{\prime}(0)=0,3 y(1)+y^{\prime}(1)=0$

where

$g(t)=\frac{-9 t^{4}+16 t^{3}+18 t+726 e^{2 t}-132 e^{t+1}\left(t^{2}+2 t+1\right)-66 e^{t}\left(2 t^{2}-7 t+11\right)+8 e^{2}\left(3 t^{4}+2 t^{3}+60 t+42\right)+e\left(15 t^{4}+32 t^{3}-228 t+540\right)+204}{24(1+e)^{2}}$

The exact solution of this problem is $y(t)=2-\frac{11 e^{t}+(3-8 e) t}{2(1+e)}$.

By applying the presented method for different values of $N$, we obtain the numerical solutions by Maple 13. Taking
$N=8,10,12$ the numerical results are shown in Table 1.

The values of $N_{e}=\left|y(t)-y_{N}(t)\right|$ at selected points. The graph of numerical solutions and absolute errors is shown in Figure 1 and 2 respectively. 
Table 1. Error analysis of Example 1 for the $x$ value

\begin{tabular}{cccccccc}
\hline $\mathrm{x}$ & Exact & & \multicolumn{5}{c}{ Present Method } \\
& Solution & $\mathrm{N}=5$ & $\mathrm{~N}_{\mathrm{e}}=5$ & $\mathrm{~N}=6$ & $\mathrm{~N}_{\mathrm{e}}=6$ & $\mathrm{~N}=7$ & \multicolumn{1}{c}{$\mathrm{N}_{\mathrm{e}}=7$} \\
\hline 0.0 & 1.000000 & 0.999999 & $0.100 \mathrm{E}-9$ & 0.999999 & $0.100 \mathrm{E}-9$ & 1.000000 & $0.000 \mathrm{E}-0$ \\
0.1 & 1.105170 & 1.105170 & $0.525 \mathrm{E}-7$ & 1.105170 & $0.341 \mathrm{E}-8$ & 1.105170 & $0.144 \mathrm{E}-9$ \\
0.2 & 1.221402 & 1.221403 & $0.274 \mathrm{E}-6$ & 1.221402 & $0.772 \mathrm{E}-8$ & 1.221402 & $0.736 \mathrm{E}-10$ \\
0.3 & 1.349858 & 1.349858 & $0.178 \mathrm{E}-6$ & 1.349858 & $0.438 \mathrm{E}-8$ & 1.349858 & $0.196 \mathrm{E}-9$ \\
0.4 & 1.491824 & 1.491824 & $0.290 \mathrm{E}-6$ & 1.491824 & $0.600 \mathrm{E}-8$ & 1.491824 & $0.490 \mathrm{E}-9$ \\
0.5 & 1.648721 & 1.648720 & $0.278 \mathrm{E}-6$ & 1.648721 & $0.270 \mathrm{E}-7$ & 1.648721 & $0.893 \mathrm{E}-9$ \\
0.6 & 1.822118 & 1.822119 & $0.110 \mathrm{E}-6$ & 1.822118 & $0.412 \mathrm{E}-7$ & 1.822118 & $0.860 \mathrm{E}-9$ \\
0.7 & 2.013752 & 2.013755 & $0.240 \mathrm{E}-5$ & 2.013752 & $0.549 \mathrm{E}-7$ & 2.013752 & $0.870 \mathrm{E}-9$ \\
0.8 & 2.225540 & 2.225536 & $0.419 \mathrm{E}-5$ & 2.225541 & $0.795 \mathrm{E}-7$ & 2.225540 & $0.596 \mathrm{E}-8$ \\
0.9 & 2.459603 & 2.459564 & $0.387 \mathrm{E}-4$ & 2.459601 & $0.117 \mathrm{E}-5$ & 2.459603 & $0.217 \mathrm{E}-8$ \\
1.0 & 2.718281 & 2.718140 & $0.141 \mathrm{E}-3$ & 2.718274 & $0.735 \mathrm{E}-5$ & 2.718281 & $0.313 \mathrm{E}-7$ \\
\hline
\end{tabular}

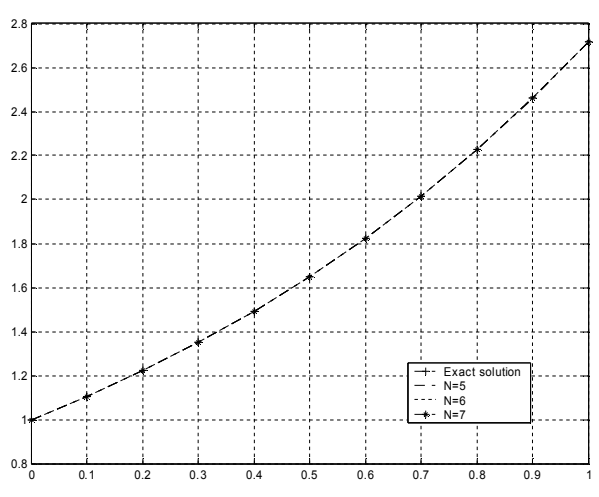

Fig.1. Numerical and exact solution of the Example 1 for $\mathrm{N}=5,6,7$.

Example 2. We consider the following problem:

$$
\begin{aligned}
& y^{\prime \prime}+y^{\prime}+y+y^{2}\left(1+y^{\prime}\right) \\
& +\int_{0}^{t}(t-s)^{2} y(s) d s=g(t)
\end{aligned}
$$

where

$$
\begin{aligned}
& g(t)=\frac{t^{4}}{4}-\frac{5(t-1)^{4}}{4} \\
& +2(t-1)^{3}+(t-1)^{2}-4 t+6
\end{aligned}
$$

with conditions

$$
2 y(0)-y^{\prime}(0)=0,3 y(1)+y^{\prime}(1)=0 \text {. }
$$

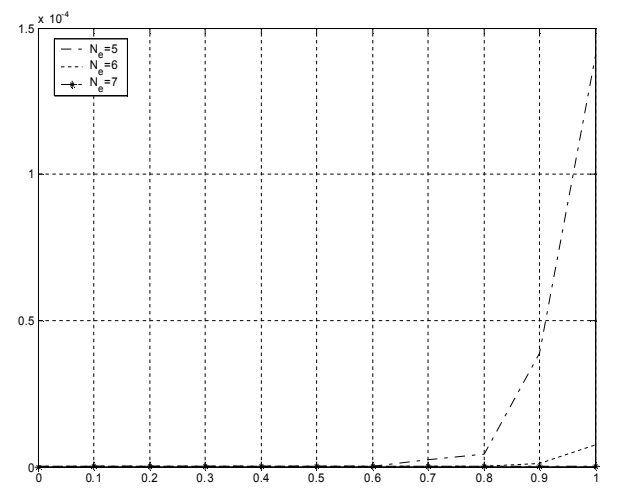

Fig.2. Error function of Example 1 for various $\mathrm{N}$.

Solving this problem for $N=4$ and we get the approximate solution $y_{4}=(t-1)^{2}$ which is the exact solution of this problem.

\section{Conclusion}

A new method for the solution of the Duffing-van der Pol equation has been proposed and investigated. The method was illustrated by accurately solving Duffing-van der Pol equations. Also, efficiency of this method has been shown in the examples. The shifted Chebyshev polynomials are used to solve the Duffingvan der Pol equation numerically. A considerable advantage of the method is that the shifted Chebyshev polynomials coefficients of the solution are found very easily by using computer programs. Shorter computation time and lower operation 
count results in reduction of cumulative truncation errors and improvement of overall accuracy. Illustrative examples are included to demonstrate the validity and applicability of the technique, and performed on the computer using a program written in Maple 9. To get the best approximating solution of the equation, we take more forms from the shifted Chebyshev expansion of functions; that is, the truncation limit $\mathrm{N}$ must be chosen large enough. $\mathrm{n}$ addition, an interesting feature of this method is to find the analytical solutions if the equation has an exact solution that is a polynomial functions. Illustrative examples with the satisfactory results are used to demonstrate the application of this method. Suggested approximations make this method very attractive and contributed to the good agreement between approximate and exact values in the numerical example.

\section{References}

Mickens, R.E., 1981. An Introduction to Nonlinear Oscillations, Cambridge Univ. Press, New York.

Guckenheimer, J., Holmes, P., 1983. Nonlinear Oscillations, Dynamical Systems, and Bifurcations of Vector Fields, Springer-Verlag, New York.

Ahmad, B., Alghamdi, B., 2007. Extended versions of quasilinearization for the forced Duffing equation. Communications on Applied Nonlinear Analysis, 4 (14), 67-75.

Tang, C.L., 1998,Solvability of the forced Duffing equation at resonance. Journal of Mathematical Analysis and Applications, 219, 110-124.

Ahmad, B., Alghamdi, B.S., 2008. Approximation of solutions of the nonlinear Duffing equation involving both integral and non-integral forcing terms with separated boundary conditions. Computer Physiscs Commucations, 179, 409-416.

Yao, H., 2009. Solution of the Duffing equation involving both integral and non-integralforcing terms. Computer Physiscs Communications, 180, 14811488.

Geng, F., 2011. Numerical solutions of Duffing equations involving both integral and non-integral forcing terms, Computer Mathematics and Applications, 61:1935-1938.

Geng, F., Cui, M., 2009. New method based on the HPM and RKHSM for solving forced Duffing equations with integral boundary conditions. Journal of Computational Applied Mathematics, 233, 165-172.

Rivlin, T.J., 1969. Introduction to the Approximation of Functions, London.

Davis, P.J., 1963. Interpolation and Approximation, Dover Publications, New York .

Body, J. P., 2000. Chebyshev and Fourier Spectral Methods, University of Michigan, New York

Atkinson, K., 2009. W. Han, Theoretical Numerical Analysis, Third Edition, Springer.

Mason, J. C., Handscomb, D. C., 2003. Chebyshev Polynomials, Chapman and Hall/CRC, New York. 